\section{Automated Functions \\ in Electron Microscopy}

\author{
Bill Tivol \\ California Institute of Technology \\ tivol@caltech.edu
}

\section{Introduction}

The newest generation of computer-controlled electron microscopes incorporates the ability to perform adjustments to microscopy conditions by comparing pairs of images and altering the conditions accordingly. Automation of electron microscope adjustments offers the advantages of accuracy, precision, efficiency, the ability to incorporate the adjustments into other automated procedures, and, for radiation-sensitive specimens, minimal exposure to the beam.

At present, automated functions include determination of eucentric height, focus, astigmatism, orientation and location of the stage tilt axis, centering of an image feature, and rotation center alignment. It is possible to automate other functions, so this list may be incomplete. In general, these functions are accomplished by induced image shifts, and, in many cases, the automated functions are completely analogous to the corresponding manual ones.

\section{Autofunctions}

Setting the eucentric height is accomplished by taking two images at different stage tilt angles, determining the image shift from a cross-correlation of the images, and using the geometric relationship between image shift and specimen height to determine the change in specimen height necessary to bring the specimen to the tilt axis (See fig. 1). The determination and setting of specimen height to about $10 \mathrm{~nm}$, reproducible to a few tens of $\mathrm{nm}$ is accomplished in about a minute with a few exposures, for which the exposed area can be minimized. These results are most reliably obtained with a thin specimen that has many distinguishable high-contrast features.

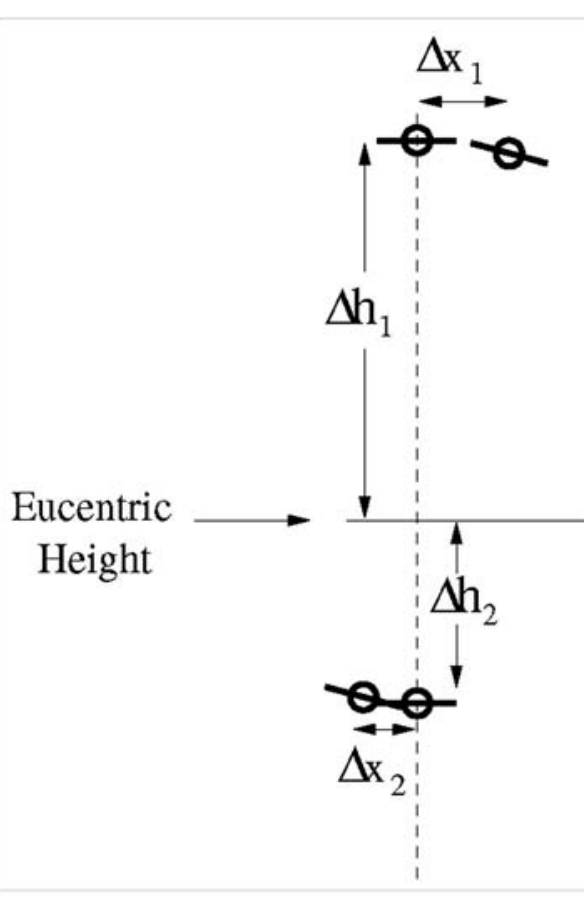

Figure 1. Illustration of the effect of tilting the stage on specimens at either of two heights offset from eucentric height.

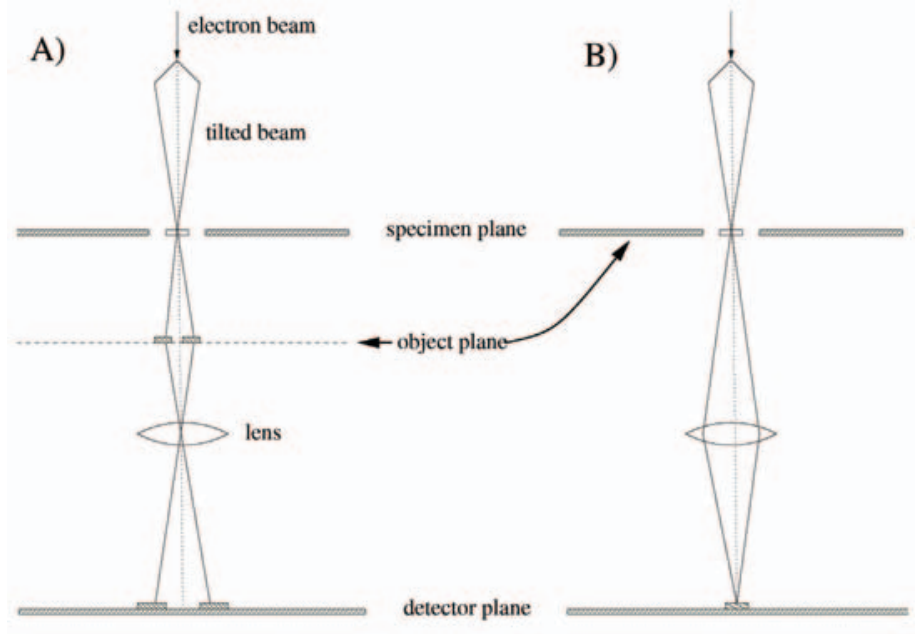

After A.J. Koster, et al., Ultramicroscopy 21 (1987) 209-222

Figure 2. Effect of beam tilt on the position of an image. A beam tilt pivot point is in the specimen plane, and the object plane is imaged onto the detector plane. A) When the microscope is not in focus, the different tilted electron beams project the specimen onto different positions on the object plane, resulting in different positions for the images on the detector plane. B) When the microscope is in focus, the object plane is coincident with the specimen plane, so the position of the image is independent of the beam tilt.

higher magnification, the accuracy and precision are better at higher magnification. With a suitable specimen the automatic procedure can determine focus to a few tens of nanometers at magnifications of a few tens of thousand times.

Astigmatism can be determined and corrected by finding the values of the defocus in two or more directions by using beam tilts with the appropriate orientations, then applying suitable currents to the stigmator coils to make the defocus value independent of direction.

Finding the location and orientation of the tilt axis is accomplished by measuring the image and focus shifts induced by stage tilts for one or more values of beam shift, then fitting those values to the expected behavior of the image as a function of distance from the axis (See Fig. 3). The accuracy and precision of this procedure depend on the reproducibility of the stage position at several tilts, so the better the eucentricity of the stage, the better the accuracy and precision will be. For the Tecnai T12 with a Gatan cryostage the results of several measurements are consistent only within several hundred $\mathrm{nm}$, and the axis position can be determined within about one hundred nm; however, with the permanently mounted stage and cartridge system on the Tecnai $\mathrm{TF} 30 \mathrm{H}$, the measurements are usually consistent within about $100 \mathrm{~nm}$, and the axis position can be determined within a few tens of $\mathrm{nm}$. This is used in different ways by tomography packages; the FEI package shifts the beam onto the tilt axis to minimize image and focus shifts during tilt-series collections, and the UCSF tomography package recalculates the offset from the tilt axis after collecting each image, then predicts the corrections in position and defocus necessary to compensate for changes from incrementing the tilt angle.

By placing the mouse pointer over a position in the image and clicking (or double-clicking) the mouse, the computer can calculate the appropriate image or stage shift required to bring that position to the center of the image. This can be used to assure that a feature of interest is centered while not exposing the specimen to a significant electron dose. It can also be used during tomography if tracking 
Don't want to miss elements that may be hiding in your sample?

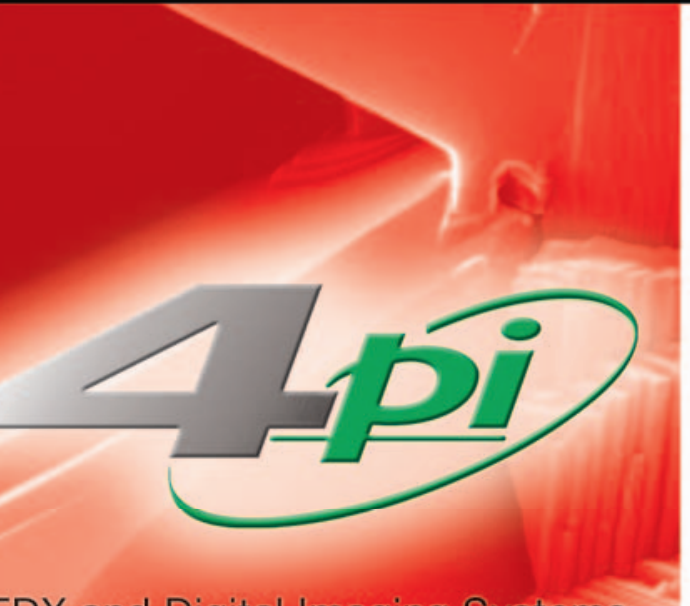

EDX and Digital Imaging Systems

\section{Maximum Pixel Spectrum}

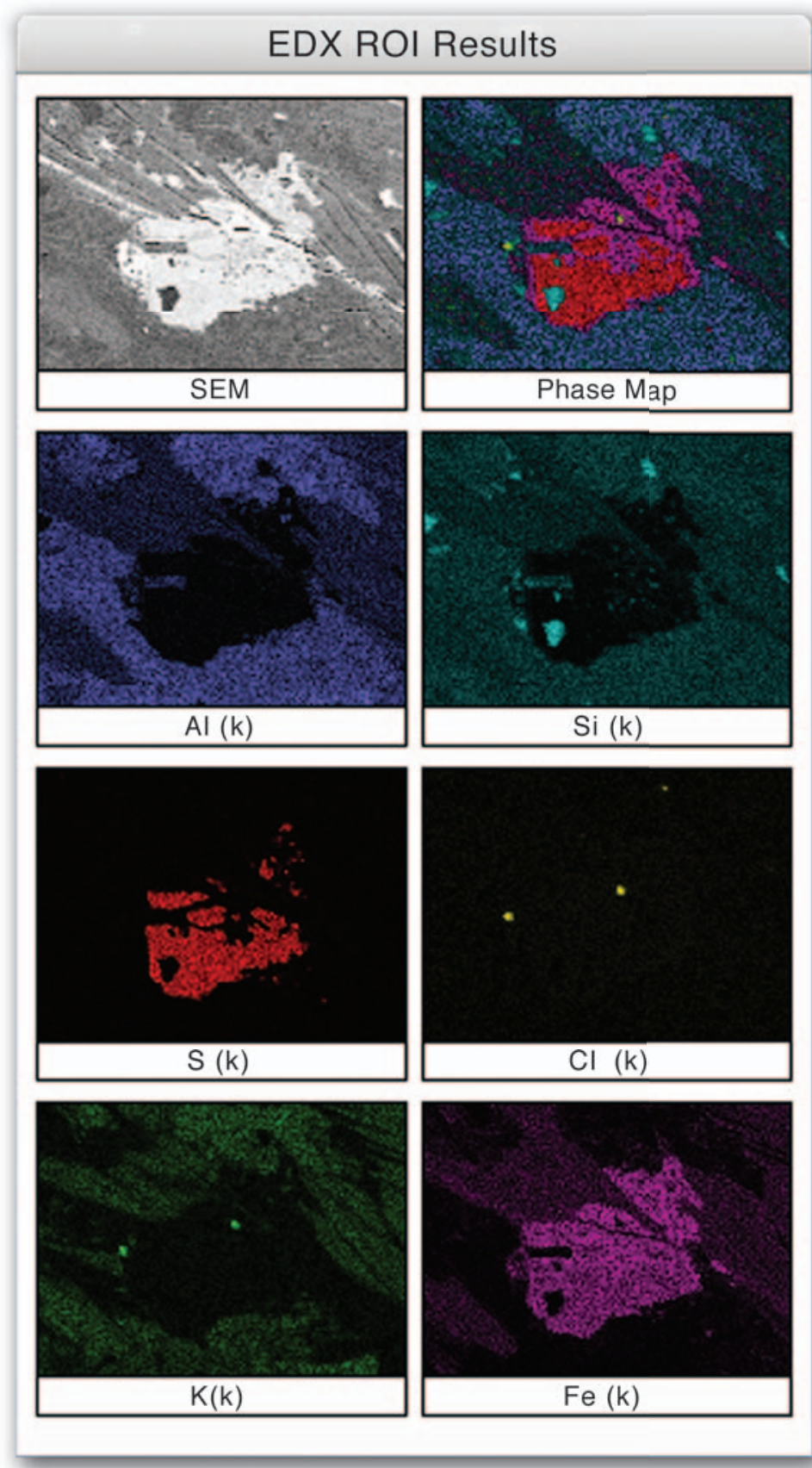

\section{joins 4pi Revolution ${ }^{\circledR}$}

Contact 4pi to ask how its Maximum Pixel Spectrum and Dynamic Element Mapping can benefit your microanalysis results.

4pi Analysis, Inc. • 919-489-1757• info@4pi.com • www.4pi.com 
"Predictive" tomography software from David Agard's group at UCSF

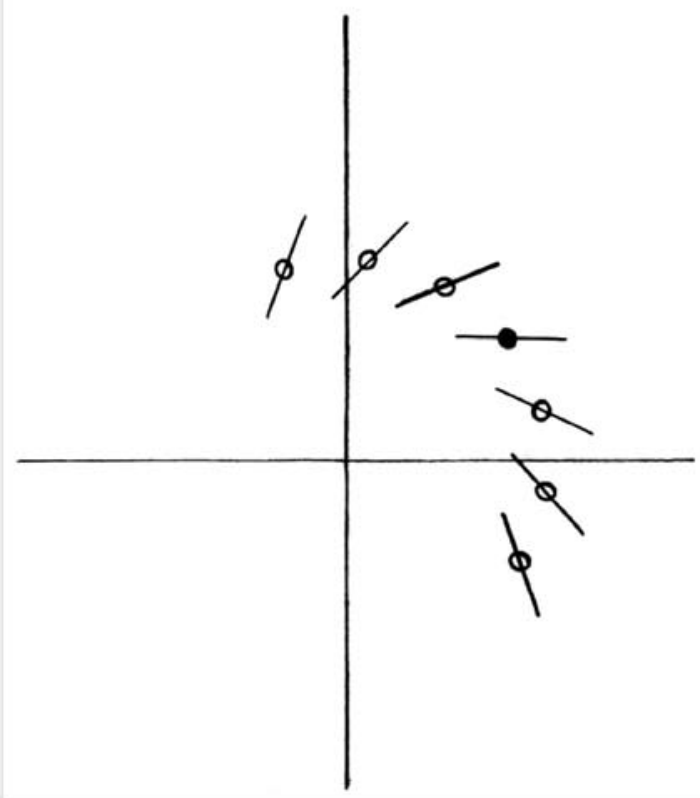

Figure 3. Motion of the specimen for a series of tilts. From measurement of the specimen position at different tilts, the position and orientation of the tilt axis can be determined; this information can be used to shift the beam onto the tilt axis, so that tracking corrections during tilting are minimized, or to predict the tracking correction for the next tilt from the tracking corrections for all the tilts obtained so far. tained at each setting can be mapped onto each other by rotation through an angle, $\theta$, about a point (the rotation center). Then the beam tilt angle can be reset so that the center of rotation is shifted to the image center (See Fig. 4). The details of how this can be accomplished are given in the Appendix.

It may also be possible to automate coma-free alignment. This is the condition for which images obtained when the beam tilt is offset by the same magnitude but in different directions have the same amount of defocus. By measuring the image defocus at several tilt offsets, using the same procedure as the autofocus function, the direction and magnitude of tilt for which all the defocus values are equal can be determined.

In addition, an automated alignment package is used to tune the imaging energy filter present on some microscopes. In the case of the Gatan Imaging Filter on the FEI Tecnai microscopes, there are seven types of alignment performed by their tuning package. These automated alignments 1) center the zero-loss peak in the energy-selecting slit (which can also be performed as a standalone function), 2) center the image onto the CCD, 3) tune the image isochromaticity to both first and 4) second order so that the electrons having a specified energy loss are all focused on the same plane-this means that either zero-loss images or element maps are equally in focus for all points on the image-5) correct chromatic aberration so that an energy shift does not shift the image, which is important for background subtraction in element maps, 6) adjust the magnification of the filter, and 7) compensate for distortions in the image.

\section{Practical Problems}

While these automated features have many advantages, it is important to realize that things can go wrong. By understanding the problems that can arise, you can learn to recognize when the features do not give the proper results. The most important thing to realize is that automated processes depend on accurate calibrations, and that none of the calibrations will be reliable unless the microscope has been accurately aligned. Proper, careful alignment is a tedious and time-consuming task, but it cannot be over-emphasized that it is essential for obtaining good results. The basic calibrations for the FEI Tecnai microscopes comprise magnification, image shift, stage shift, and focus.

The magnification calibration is determined by inserting a cross-grating and calculating the spatial frequency of the repeat. This method gives the repeat distance averaged over as many squares as are present in the field of view of the CCD camera, which is more accurate than simply measuring the distance between one grating line and another. However, in order to get an accurate measure, the grid must be at the eucentric height and in focus (both set manually at this point). Also the grid must be flat and untilted, and must not be distorted. A particular magnification is automatically selected by the software. Optimal magnification for this calibration is a balance between having a large number of grating squares in the field of view and having as many pixels as possible between grating lines.

Image shift calibrations are accomplished by applying a particular excitation to the shift coils and measuring the corresponding change in the image using cross-correlation. This procedure must

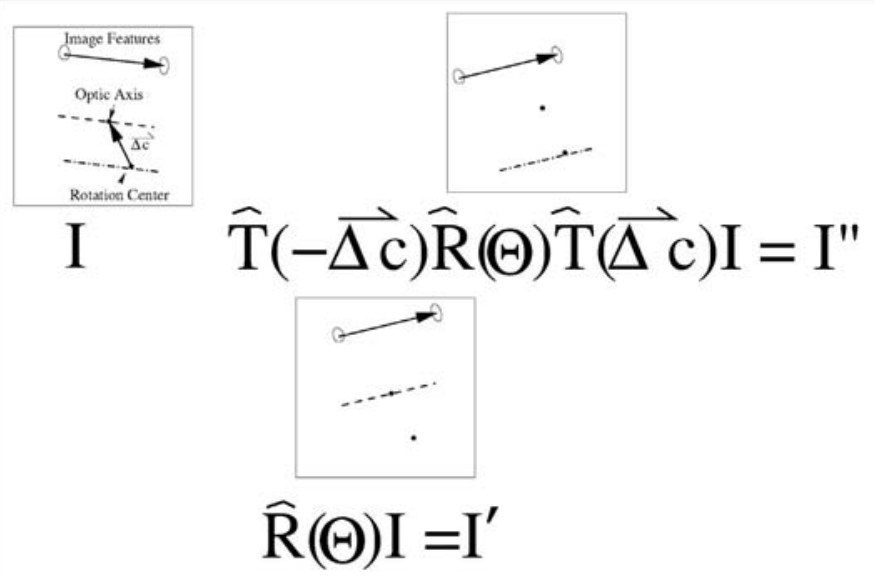

Figure 4. Illustration that rotation of an image about a point that is offset from the center of the image is equivalent to rotation of the image about its center plus a translation. The initial image, I, includes features, two of which are shown with the vector between them. Also shown are the optic axis at the origin of the image coordinate system, the rotation center displaced from the optic axis by the vector $\overrightarrow{\Delta c}$, and lines parallel to the vector between image features and passing through the optic axis and the rotation center. The image I' was produced from image I by a rotation through an angle $\theta$ about the optic axis. Note that the vector between the features is unchanged in magnitude and is still oriented parallel to the line through the optic axis. The image I" was produced from image I by a rotation through an angle $\theta$ about the rotation center. Note that the vector between the features is unchanged in magnitude and is still oriented parallel to the line through the rotation center. The equations underneath the images express the fact that rotation about the rotation center is equivalent to translation of the image to place the rotation center onto the optic axis, rotation about the optic axis, then translation of the image to return the rotation center to its original position. Note that the vectors between the features in I' and I" have both the same magnitude and orientation, so the two images must be related by a translation. See the appendix. 


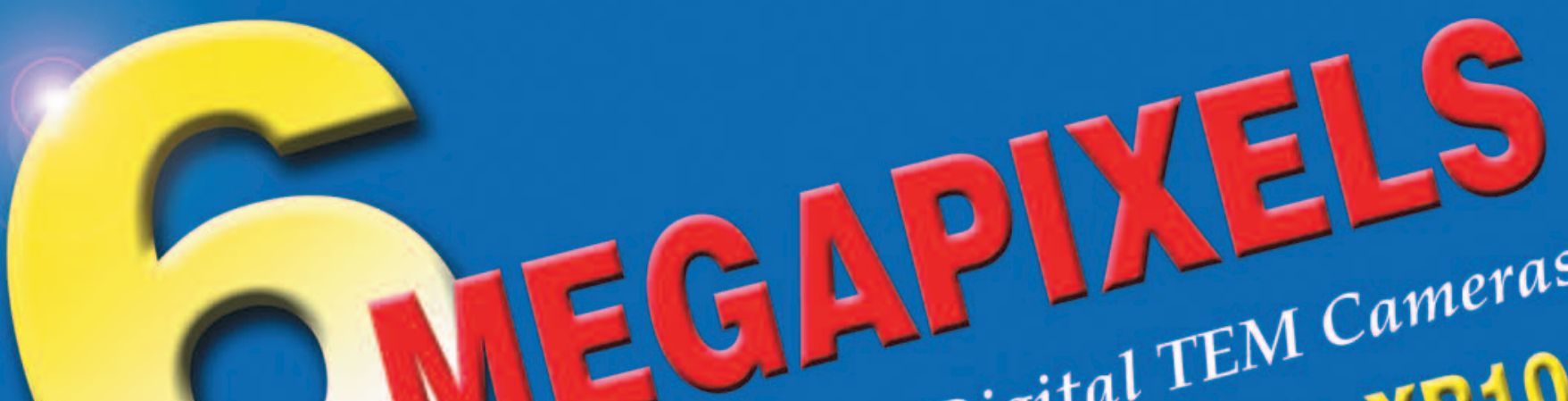
High Definition Digi

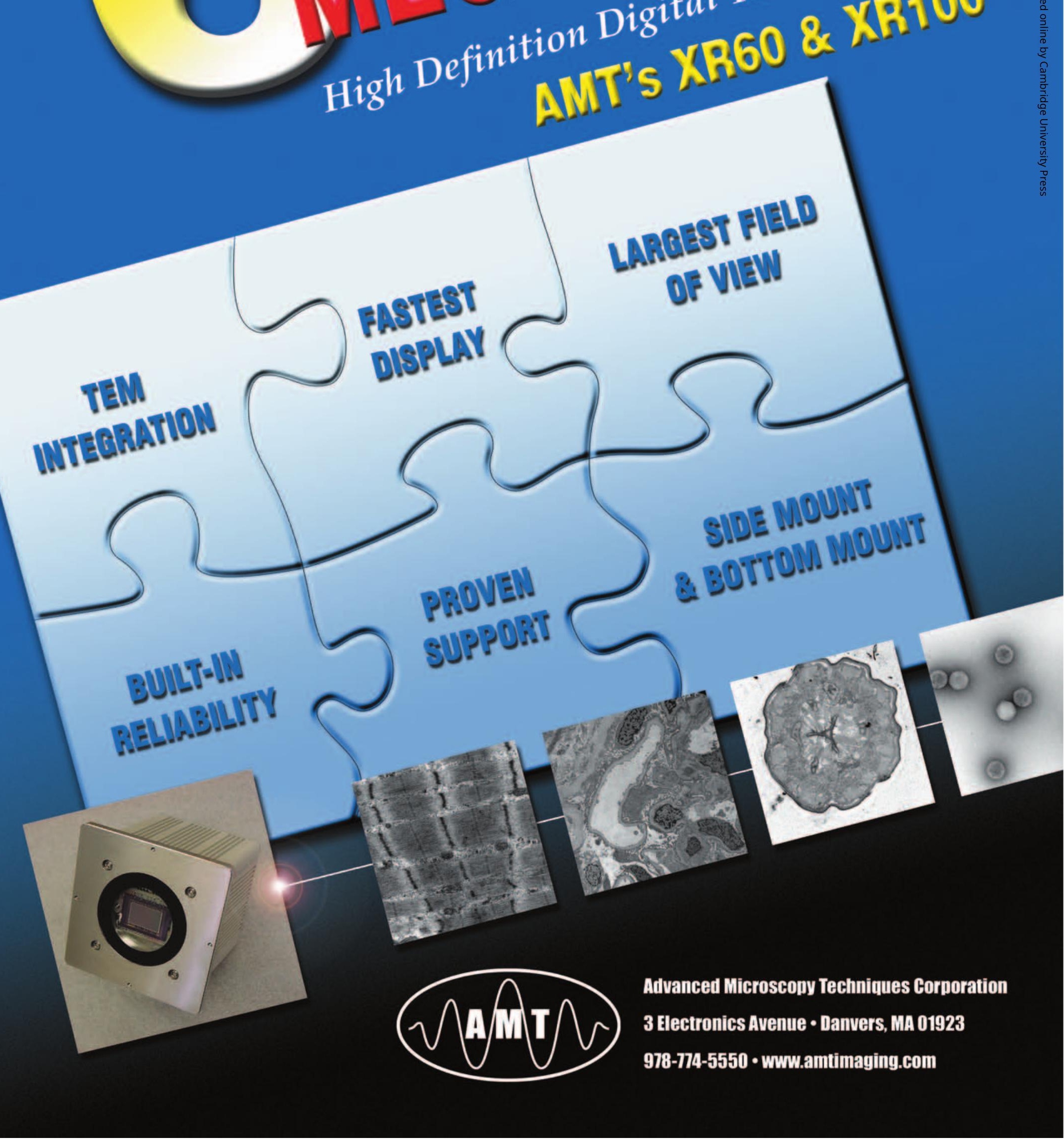


be done at each magnification for which autofunctions will be used. Obviously, anything other than shift coils that affects the position of the image will interfere with this calibration, so drift, changes in the specimen due to heating or charging, etc., must be eliminated.

The stage shift calibration connects the physical motion of the stage with an excitation of the image shift coils that has the same effect. In this way the physical motion of the stage, the excitation of shift coils and the change in the image, measured in pixels, are linked with one another. Once again, stage or specimen drift will interfere with this calibration.

Focus calibration determines the image shift induced by two beam tilts of a specific magnitude and in opposite directions; this procedure is performed at different values of defocus. Since the relationship between beam tilt and image shift is not linear, automatic focusing should be performed using the same magnitude of beam tilt as was used in the calibration. As usual, anything that causes the specimen or image to shift (other than the expected beam-tilt-induced image shift) will cause problems. Using a thin, robust, high-contrast specimen that is a good conductor of heat and electricity is essential; a distribution of colloidal gold spheres on a thin carbon film meets all the requirements.

Since cross-correlation between two images is the technique used to calibrate and determine essentially all the automatically measured parameters, anything that renders that process unreliable will have severe adverse effects on autofunctions. One obvious problem for cross-correlation is that the two images being compared must have sufficient features in common. That means that the induced image shifts must be significantly smaller than the length of the image.

Furthermore, it is often advantageous to use a center-weighted filter, such as a Hanning window, to get good cross-correlation results. Cross-correlations are performed by assuming that the images repeat to fill space, so if there is a gradient of intensity across the image, this leads to a step in intensity at the edges of the original image, and this step can easily be the most prominent feature in the image, so it will dominate the cross-correlation. In the case that there are intensity differences at both the horizontal and vertical edges, the cross-correlation will have a large false peak in the center. By multiplying the original intensities by a function that is a cosine plus a constant (so that it is 1 in the center and 0 at the edges), this false peak is eliminated, but now the image cannot shift so far that centrally-located features in the unshifted image are damped out by the filter in the shifted image.

Noisy images and those without unique prominent features can give ambiguous cross-correlations. The occurrence of patterns of noise in one image that happen to fit patterns in the second image will give a false peak, and, if there are only subtle intensity differences in each image, such as those in low-dose, frozen-hydrated, unstained, close-to-focus images of biological material, a random peak may be high enough to predominate over real correlations.

Even with a thin, high-contrast specimen, the autofunctions can give surprising results. For example, if you insert a gold-onthin-carbon grid, set it to eucentric height (either manually or automatically), and perform the autofocus several times, the focus that is found will oscillate with decreasing amplitude and almost always converge at a value that is consistent. Thus, if one accepts the first value found, the focus will not, in general, be correct.
If the specimen is less than ideal, there are greater problems. The previously-mentioned frozen-hydrated specimens can be especially troublesome, and the best way to assure that there are areas of good contrast is to deposit gold beads onto the support film before freezing the specimen. The beads should be large enough to cover several pixels, so the optimal size depends on the magnification. If the image magnification is to be different from that used for focusing, and if more than one image is to be aligned using gold beads as fiducial markers, it will be best if the beads used for focusing are put on the support film and those for alignment are mixed with the specimen prior to freezing. Another problematic specimen is one with high contrast, but thick. In this circumstance, the most prominent feature could be at any depth in the specimen, and the image shift measured by cross-correlation could be determined by one (or a few) features or could be a compromise over all the features in the specimen. As a result, the autofunctions could pick out different planes within the specimen to set the eucentric height, focus, etc.

Although this list of problems with autofunctions seems long, the advantages of autofunctions far outweigh the difficulties. However, knowledge of the kinds of problems that can occur indicate the need to watch the results to detect when something has gone wrong, rather than simply assuming that autofunctions will always work perfectly.

\section{APPENDIX}

Figure 4 shows the relationships among vectors involving the optic axis, the rotation center, an arbitrary point in the image, $(x, y)$, and the changes in $(x, y)$ resulting from rotation through an angle, $\theta$, about either the optic axis, $\overrightarrow{\Delta r_{1}}$, or the rotation center, $\overrightarrow{\Delta r_{2}}$. Comparison between images I' and I" (see Fig. 3 ) will show a displacement, $\vec{\tau}$, equal to the difference $\overrightarrow{\Delta r_{2}}-\overrightarrow{\Delta r_{1}}$. Also shown in the figure is an enlargement of the relevant area giving the magnitudes and orientations of $\overrightarrow{\Delta r_{1}}$ and $\overrightarrow{\Delta r_{2}}$. The rotation operator can be written in matrix form, which makes clear that it is linear:

$$
\bar{R}\left(\frac{\pi+\theta}{2}\right)=\left(\begin{array}{cc}
\cos \left(\frac{\pi+\theta}{2}\right) & \sin \left(\frac{\pi+\theta}{2}\right) \\
-\sin \left(\frac{\pi+\theta}{2}\right) & \cos \left(\frac{\pi+\theta}{2}\right)
\end{array}\right)
$$

Then we have, $\overrightarrow{\Delta r_{1}}=2 \sin \frac{\theta}{2} \bar{R}\left(\frac{\pi+\theta}{2}\right) \overrightarrow{r_{1}}, \overrightarrow{\Delta r_{2}}=2 \sin \frac{\theta}{2} \bar{R}\left(\frac{\pi+\theta}{2}\right) \overrightarrow{r_{2}}$,

and, since $\overrightarrow{\Delta c}+\vec{r}_{1}=\vec{r}_{2}$, we get

$$
\vec{\tau}=\overrightarrow{\Delta r_{2}}-\overrightarrow{\Delta r_{1}}=2 \sin \frac{\theta}{2} \bar{R}\left(\frac{\pi+\theta}{2}\right)\left(\overrightarrow{r_{2}}-\overrightarrow{r_{1}}\right)=2 \sin \frac{\theta}{2} \bar{R}\left(\frac{\pi+\theta}{2}\right) \overrightarrow{\Delta c},
$$

and therefore,

$$
\overrightarrow{\Delta c}=\bar{R}\left(-\frac{\pi+\theta}{2}\right) \frac{\vec{\tau}}{2 \sin \frac{\theta}{2}}=\frac{1}{2 \sin \frac{\theta}{2}}\left(\begin{array}{cc}
\cos \left(\frac{\pi+\theta}{2}\right) & -\sin \left(\frac{\pi+\theta}{2}\right) \\
\sin \left(\frac{\pi+\theta}{2}\right) & \cos \left(\frac{\pi+\theta}{2}\right)
\end{array}\right) \vec{\tau},
$$

which determines the correction necessary to shift the rotation center onto the optic axis from the displacement that maps image I" onto image I'. 


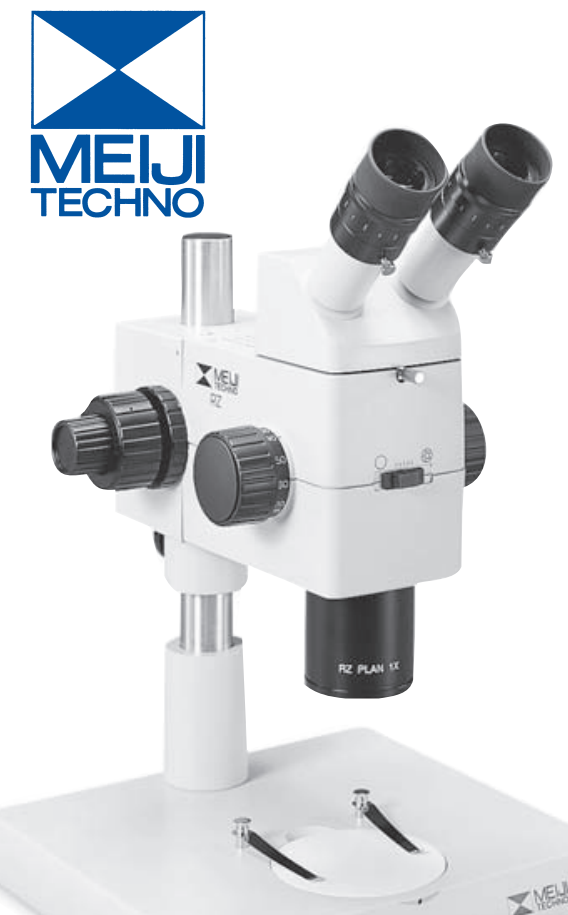

\section{The Meiji RZ Series of Research Stereo Microscopes.}

If you are looking for precision, durability, quality and value in a high performance Stereo Microscope, we invite you to take a closer look at Meiji's RZ Series of Research Stereo Microscopes.

The RZ Series modular system design allows you the freedom to create an ideal instrument for your specific need or application. Featuring a 10:1 zoom ratio, variable double iris diaphragm, and positive detente click stops at 12 positions of magnification. A full range of optional accessories is available, including: Video and photo-micrographic systems, brightfield-darkfield transmitted light stands, ergonomic binocular head, drawing attachment, multiple interchangeable objectives and wide-field eyepieces. Complete system versatility backed by a "Limited Lifetime Warranty."

For more information on these economically priced Stereo Microscopes, please call, FAX, write us or log on to our website today.

\section{MEIJI TECHNO AMERICA}

2186 Bering Drive, San Jose, CA 95131,

Tel: 408.428.9654, FAX: 408.428.0472

Toll Free Telephone: 800.832 .0060 or visit our website at www.meijitechno.com

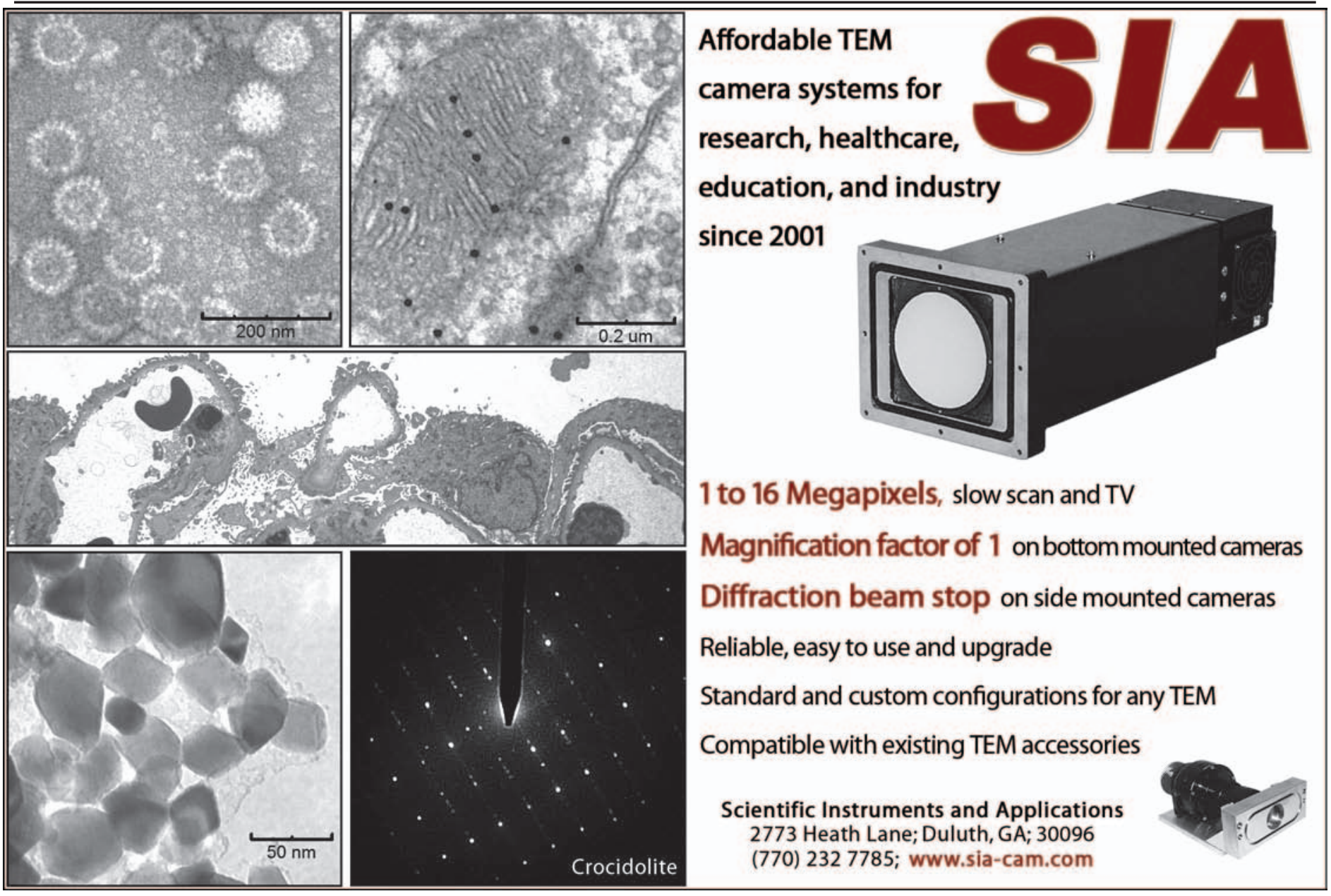

